\title{
Parathyroidectomy May Cause Remission of Uraemic Tumoral Calcinosis in Haemodialysis Patients
}

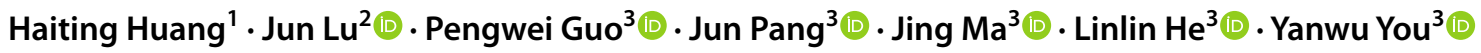

Received: 20 October 2021 / Accepted: 31 December 2021

(C) The Author(s) 2022

\begin{abstract}
Few cases of uraemic tumoral calcinosis (UTC) have been reported. This study aimed to investigate the clinical efficacy of parathyroidectomy for UTC. Historical clinical data of patients with end-stage renal disease and UTC who underwent parathyroidectomy were analysed. Absorption of metastatic calcification was compared before and after operation. Changes in intact parathyroid hormone, serum calcium, phosphorus, and alkaline phosphatase levels were analysed before parathyroidectomy and at 1 week and 3, 6, and 12 months after parathyroidectomy. Eight patients met the enrolment criteria (men, 6; mean age, 38.6 SD 10.9 years). Uraemic tumoral calcinosis, which developed $2-8$ years after dialysis began, was caused by secondary hyperparathyroidism. Massive calcium deposition was found in the shoulder $(n=6)$, hip $(n=3)$, and elbow $(n=2)$. Four patients had $>2$ joints affected, and a single joint was involved for four patients. Seven patients had rapid remission ( $<6$ months) of the masses after parathyroidectomy. In one patient, the mass remained unabsorbed until 6 months postoperatively. Hypocalcaemia occurred in all patients where parathyroidectomy was successful, and calcium supplementation was required 1 year postoperatively. Serum intact parathyroid hormone levels on day 7 and at 3 and 6 months postoperatively decreased significantly from baseline and remained low 1 year postoperatively $(22.015 \mathrm{SD} 33.134 \mathrm{pg} / \mathrm{mL})$. Postoperative phosphorus levels were significantly lower than preoperative levels $(p<0.05)$, but no significant difference was found in alkaline phosphatase levels $(p>0.05)$. Parathyroidectomy has promising efficacy for UTC treatment and regulation of serum intact parathyroid hormone and phosphorus. Hypocalcaemia is a common complication after parathyroidectomy. Current Controlled Trials ChiCTR2000041311, date of registration: Dec. 23, 2020.
\end{abstract}

Keywords Secondary hyperparathyroidism $\cdot$ Parathyroidectomy $\cdot$ Tumoral calcinosis $\cdot$ Haemodialysis

\section{Introduction}

Uraemic tumoral calcinosis (UTC), a disease of abnormal bone and mineral metabolism, is a rare complication of endstage renal disease (ESRD) that causes abnormal deposition of calcium and phosphorus around large articular structures.

Jun Lu And Pengwei Guo are co-first authors

Yanwu You

youyanwu@163.com

1 Department of Nephrology, The First Affiliated Hospital of Jinan University, Guangzhou, Guangdong Province, China

2 Medical Imaging Experiment and Training Center, Youjiang Medical University for Nationalities, Baise, Guangxi, China

3 Department of Nephrology, Youjiang Medical University for Nationalities, No. 18 Zhongshan Road II, Baise 533000, Guangxi, China
Most patients are asymptomatic for several years, although some may experience skin ulcerations and joint movement and activity limitations [1].

Little is known about its exact mechanism, and limited treatments show varied efficacies. Most researchers believe that UTC is usually caused by hypercalcaemia and hyperphosphataemia resulting from secondary hyperparathyroidism (SHPT). UTC typically occurs 8-36 months after the onset of SHPT, with morbidity rate of $0.5-3 \%$ in patients receiving haemodialysis [2]. Treatment strategies for UTC include reduction of the $\mathrm{Ca} \times \mathrm{P}$ product and contributors to mineralisation [3], kidney transplantation, or parathyroidectomy (PTX). Parathyroidectomy is considered effective for the treatment of UTC in haemodialysis patients with SHPT. In some UTC patients, however, PTX is ineffective [4]. This study aimed to investigate the clinical efficacy of PTX to treat UTC in eight patients to provide a reference for clinical practice. 


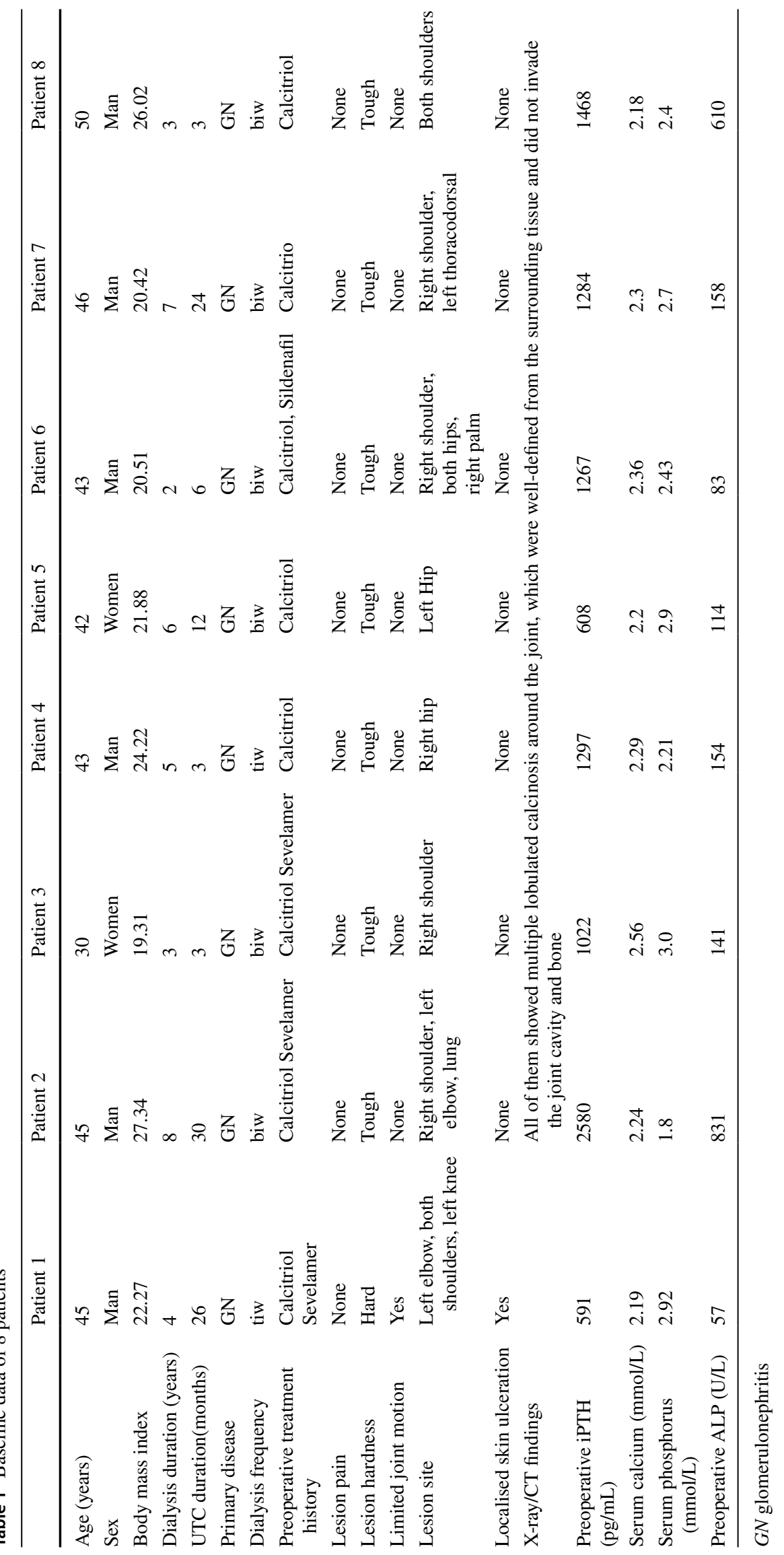




\section{Patients and Methods}

\section{Study Population}

Patients with SHPT and UTC who underwent maintenance haemodialysis in our dialysis centre and underwent total PTX without transplantation (tPTX) between January 2008 and January 2019 were eligible for inclusion. The inclusion criteria were diagnosis of SHPT with tumour-like metastatic calcification and PTX. The exclusion criteria were SHPT with a history of drug treatment and history of multiple neck operations. Clinical data, including age, sex, body mass index (weight/height; $\mathrm{kg} / \mathrm{m}^{2}$ ), primary disease, dialysis method, dialysis duration, metastatic calcification duration, and degree and time of postoperative absorption of metastatic calcinosis, and levels of intact parathyroid hormone (iPTH), serum calcium, phosphorus, and alkaline phosphatase (ALP) before and after surgery, were collected. Informed consent was obtained from all the participants. This study was approved by the human research ethics committee of the Affiliated Hospital of Youjiang Medical College for Nationalities (YYFY-LL-2019-004).

\section{Biochemical Examination}

Intact parathyroid hormone, serum calcium, serum phosphorus, and ALP levels at 1 week and 3, 6, and 12 months after surgery were measured. Levels of iPTH (normal, 6-80 pg/mL) were measured using enzyme-linked immunosorbent assay. Serum calcium (normal, 2.02-2.6 mmol/L), serum phosphorus (normal, 0.81-1.62 mmol/L), and ALP (normal, 31-135 U/L) levels were measured using a Japanese 7600-010 Blood Biochemical Automatic Analyser (Roche Diagnostics). Patients 4 and 7 failed to return to our dialysis centre for postoperative re-examination due to family circumstances; thus, the results of the blood draws and re-examinations were obtained from the records of the local dialysis centres where the procedures were performed.

\section{Image Analysis}

X-ray or CT scans were performed 3, 6, and 12 months after the operations to compare the absorption of metastatic calcinosis at each time point. Two senior imaging physicians analysed the images independently. The patchy, lumpy, and cluster calcification in soft tissues and the calcification area of soft tissues were calculated.

\section{Surgical Methods}

All procedures were performed by the same gland surgery team. Given the current best evidence, together with our clinical experience and the attitudes of the patient and family members, general anaesthesia, cervical transverse incisions, and tPTX were performed in all eight patients. All resected glands were sent to the Department of Pathology of our hospital for histopathological examination.

\section{Evaluation Index and Related Definition}

Secondary hyperparathyroidism was diagnosed when the patient's serum iPTH was $>800 \mathrm{pg} / \mathrm{mL}$ (continuous refers to more than two test results). Surgical success was defined as $\mathrm{iPTH}<300 \mathrm{pg} / \mathrm{mL}$ in the first postoperative week. The phenomenon of extraosseous calcium deposition in the soft tissue around the joint in patients with end-stage renal disease is known UTC. The duration of UTC was defined as the time interval between the first discovery of the tumour and day of operation. The UTC size was defined as the size of the tumour protruding from the skin surface. With regard to the degree of UTC absorption, the tumour size and calcification area were reduced by more than half postoperatively, which was defined as obvious absorption. Complete absorption occurred if the tumour disappeared completely. The duration of dialysis covered the beginning of dialysis to the diagnosis.

\section{Postoperative Calcium Supplementation}

On the first postoperative day, after conventional administration of oral calcitriol ( $2 \mu \mathrm{g} \mathrm{QN})$ and calcium bicarbonate (1.5 g TID), each patient received calcium gluconate injection, which was continuously pumped with

Table 2 Features of uraemic tumoral calcinosis and comparison of absorption lesions after operation

\begin{tabular}{|c|c|c|c|c|c|}
\hline Patient no & $\begin{array}{l}\text { UTC duration } \\
\text { (months) }\end{array}$ & $\begin{array}{l}\text { Postoperative absorption } \\
\text { time (months) }\end{array}$ & Lesion location & $\begin{array}{l}\text { W/wot postoperative } \\
\text { recurrence }\end{array}$ & Pathology \\
\hline Patient 1 & 26 & 12 & Left elbow, both shoulders, left knee & Yes & Hyperplasia \\
\hline Patient 2 & 30 & 6 & Right shoulder, left elbow, lung & None & Adenoma \\
\hline Patient 3 & 3 & 1 & Right shoulder & None & Adenoma \\
\hline Patient 4 & 3 & 2 & Right hip & None & Adenoma \\
\hline Patient 5 & 12 & 2 & Left hip & None & Adenoma \\
\hline Patient 6 & 6 & 2 & Right shoulder, both hips, right palm & None & Hyperplasia \\
\hline Patient 7 & 24 & 5 & Right shoulder, left thoracodorsal & None & Adenoma \\
\hline Patient 8 & 3 & 1 & Both shoulders & None & Hyperplasia \\
\hline
\end{tabular}



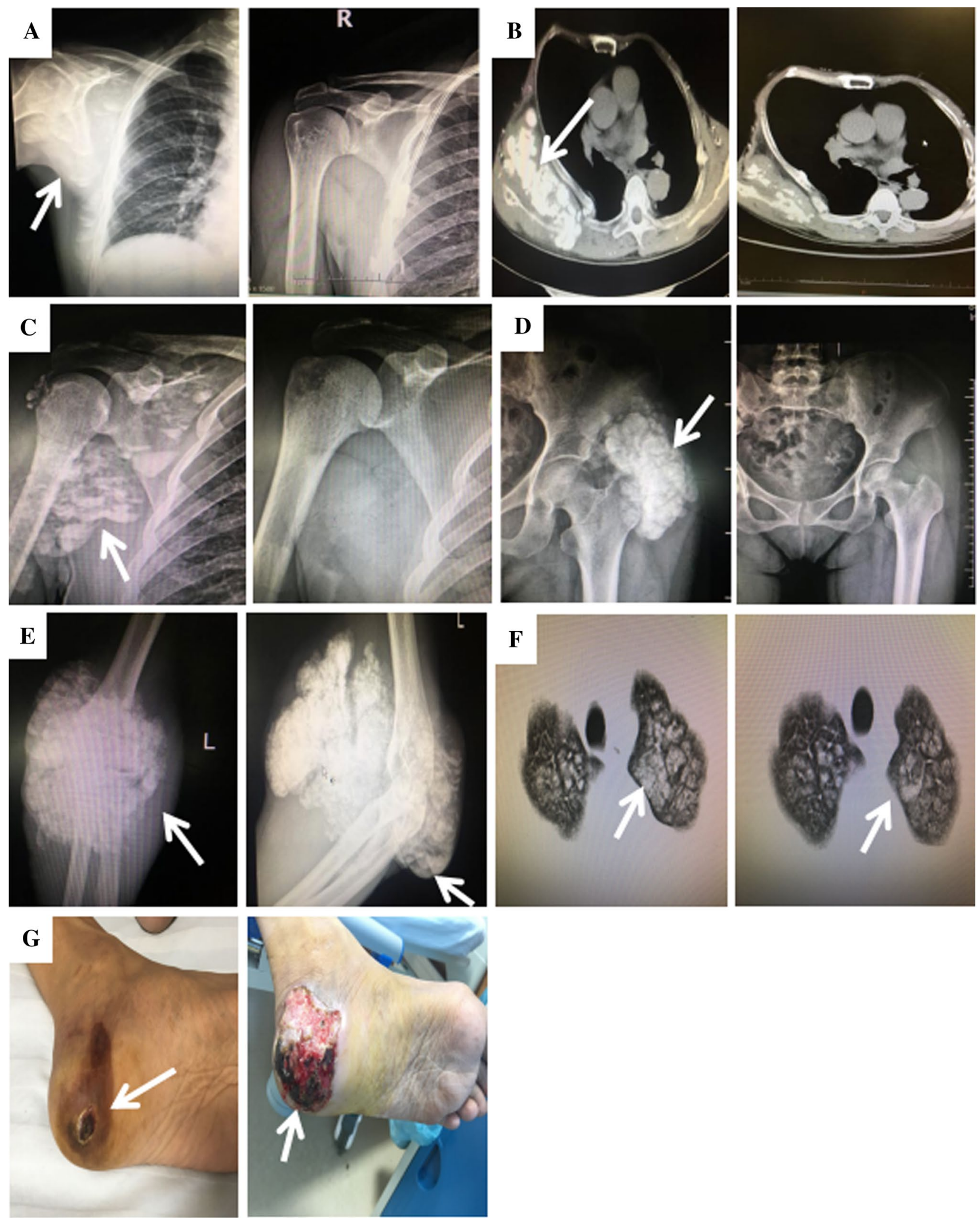
४Fig. 1 Imaging comparison of uraemic tumoral calcinosis before and after PTX. In patient 1, the lesion of the right shoulder joint was completely absorbed 2 months after PTX (A). In patient 2, the lesion of the right shoulder joint was mostly absorbed 6 months after PTX (B). In patient 3, the lesion of the right shoulder joint was completely absorbed 1 month after PTX (C). In patient 5, the lesion of the left hip joint was completely absorbed 2 months after PTX (D). In patient 1 , the lesion density was further increased at 12 months after PTX (E). In patient 2, metastatic pulmonary calcifications showed remarkable absorption on imagery 6 months after PTX (F). In patient 2, the calciphylaxis on the left heel had progressed significantly 6 months after PTX (G)

a micropump at $2 \mathrm{mg} /(\mathrm{kg} / \mathrm{h})$. Serum calcium levels were evaluated every $6 \mathrm{~h}$ on the first postoperative day, and once a day on postoperative days 2-7. Intravenous calcium supplementation was terminated once the serum calcium concentration was $>2.8 \mathrm{mmol} / \mathrm{L}$. Calcitriol and calcium bicarbonate doses were then halved, and the serum calcium concentration was re-evaluated once a month to adjust the oral calcium dose.

\section{Statistical Analysis}

All data were processed using SPSS 13.0 statistical software (IBM Corp., Armonk, NY, USA). Measurement data with normal distribution were expressed as mean and standard deviation, and measurement data with nonnormal distribution were expressed as $m(1 / 4,3 / 4)$. When the measurement data were consistent with the homogeneity of variance, paired $t$-test was used for comparison between groups. Statistical significance was accepted when $p<0.05$.

\section{Results}

\section{Baseline Data}

From January 2008 to January 2019, there were 519 maintenance haemodialysis patients in our dialysis centre, among which 205 (39.4\%) patients developed SHPT and 10 (1.92\%) patients had SHPT combined with UTC. Eight patients met the inclusion criteria of this study (among the two patients who did not meet the inclusion criteria, one patient underwent local resection of the shoulder joint tumour; despite wound healing after operation, the patient died of septic shock. The other patient's buttock tumour subsided after oral administration of Sevelamer Carbonate Tablets for 2 months).

We enrolled eight patients (men, 6; aged 45-60 years, women, 2; aged 30-42 years) with SHPT and UTC. The primary disease was chronic glomerulonephritis for all eight patients. The dialysis duration was $2-8$ (average, 4.75 SD 2.12) years. The
UTC duration was 3-30 (average, 13.37 SD 11.51) months. At disease onset, all patients presented with a painless mass, and the surface of the mass ruptured, with chronic sinus formation occurring 1 year after onset in patient 1 . The shoulder $(n=6)$, hip ( $n=3)$, and elbow $(n=2)$ were involved, and patients had a single affected joint $(n=4)$ or $>2$ affected joints $(n=4)$. Patient 2 had pulmonary metastatic calcification and calciphylaxis of the left heel. No patient had a history of trauma or surgery at disease site. Baseline data are compared in Table 1.

\section{Surgical Results}

All patients underwent tPTX, and seven of the surgeries were successful. In patient 4 , only three parathyroid glands were found. One week after surgery, the iPTH level for this patient was still $>300 \mathrm{pg} / \mathrm{mL}$, which did not meet the criteria for surgical success. The pathological types of the parathyroid glands were adenoma $(n=5)$ and hyperplasia $(n=3)$ (Table 2).

\section{Postoperative Absorption of UTC}

The postoperative absorption of lesions in eight patients is detailed in Table 2. Nearly complete absorption of the lesions at the joint sites was observed in seven patients 1-6 months after surgery (Fig. 1A-D), including patient 4 for whom the surgery was not surgically successful. At 6 months after surgery, patient 1 had slightly reduced metastatic calcification in the elbow joint, but at 12 months, the patient presented with an even greater density of metastatic calcinosis in the left elbow joint and a mass, which developed postoperatively (Fig. 1E). Patient 2 had pulmonary metastatic calcification and calciphylaxis of the left heel; the number of metastatic pulmonary calcifications significantly reduced 6 months after operation (Fig. 1F), but calciphylaxis on his left heel worsened (Fig. 1G). Symptoms of skin itching, bone pain, and insomnia were significantly improved in all patients. The blood pressure of patients with intractable hypertension before surgery was well controlled postoperatively.

\section{Dynamic Monitoring of Serum iPTH, ALP, Calcium, and Phosphate Levels After PTX}

Preoperative and postoperative biochemical indicators are compared in Table 3. In the successful surgery group, the serum iPTH level decreased significantly by day 7 and remained low at 3,6 , and 12 months after surgery $(p<0.001)$. In patient 4 , the iPTH level gradually increased to preoperative levels 6 months after surgery. All seven patients whose surgery was successful developed hypocalcaemia, and they required long-term oral calcitriol and calcium carbonate treatments. Subsequently, the serum calcium levels 
in these patients dropped and were maintained at $1.70 \mathrm{SD}$ $0.22 \mathrm{mmol} / \mathrm{L}$ at 1 year after surgery, and hypocalcaemia symptoms disappeared. The serum phosphorus levels of these patients also decreased significantly after surgery $(p<0.05)$, whereas ALP levels did not change significantly $(p>0.05)$.

\section{Discussion}

A total of eight patients who had metastatic calcinosis at various joint and non-joint sites (i.e. chest wall), including the shoulder joint (5/8), hip joint (3/8), and elbow joint $(2 / 8)$, were included in this study. The predilection sites were consistent with those in previous studies [1]. It is unclear why UTC occurs frequently at large joints. Fukuta et al. [5] found that in patients with SHPT, pulmonary calcinosis often occurred in the upper lung, and onset was related to the presence of a $\mathrm{pH}$ gradient in the lung tissue. The $\mathrm{pCO}_{2}$ in the upper lung was lower than that in the lower lung, and the $\mathrm{pH}$ was alkaline, which resulted in calcium phosphate precipitation. This suggests that the onset of UTC in large joints may be related to a change in the $\mathrm{pH}$ of synovial fluid, which is similar to the appearance of tophi in the joints of individuals with gouty arthritis. However, no studies have examined the correlation between the change in $\mathrm{pH}$ in the synovial fluid of patients with SHPT and the occurrence and progression of UTC. Uraemic tumoral calcinosis often appears 8-36 months after SHPT onset, with an incidence rate of $0.5-3.0 \%[6,7]$, and the actual incidence rate may be higher due to the lack of specific symptoms at the early disease stage. With the advancements in blood purification technologies, the incidence of SHPT is increasing annually, and the incidence of UTC increases accordingly [8].

A diagnosis of UTC mainly relies on the patient history and typical imaging findings. The primary clinical manifestation of UTC is a painless mass in the periarticular soft tissues that does not involve the synovium of the joint, and this slowly growing mass can lead to limited joint function. Uraemic tumoral calcinosis presents as subcutaneous papules or nodules in the skin, with no significant visible changes in the local skin, in which excessive tumours can form a sinus with white silt-like material flowing from the skin opening. Radiography, CT, and magnetic resonance imaging are highly sensitive and can show irregular lobulated hyperdense calcinosis without destruction of the periarticular bone $[9,10]$. In this study, microscopically, amorphous calcium surrounded by dense fibrous tissue was observed, and the main components were hydroxyapatite and calcium pyrophosphate dihydrate crystals. An infiltration of macrophages, fibroblasts, and osteoclastlike giant cells was also observed in some cases. All eight patients had a painless mass at a large joint site as the first symptom. One patient had UTC for more than 30 months, the surface of the mass ruptured, and a chronic sinus formed. The texture became exceptionally hard, which was consistent with typical clinical manifestations of UTC.

The pathogenesis of UTC has not been elucidated, and some researchers believe that the disease is caused by autosomal dominant genetic malformation $[3,11]$. Other researchers consider that a primary defect of the collagen tissue causes a stress response of the collagen fibres around the diseased joint [12]. Some scholars believe that the abnormal metabolism of calcium, phosphorus, and cholesterol results in the ectopic distribution of calcium [13]. Vieira et al. [14] also suggested that the disease was caused by calcium redistribution due to a local nutrient supply disturbance following trauma. At present, scholars mainly agree with the theory of abnormal calcium and phosphorus metabolism. Several UTC cases with a history of calcitriol pulse therapy have been reported [15, 16]. In the present study, eight patients had a long-term history of taking Calcitriol. Therefore, we believe that excessive intake of active vitamin D3 may be the main reason for the development of UTC. The primary disease of the eight patients with chronic renal failure was primary

Table 3 Dynamic changes of iPTH, Ca, P, and ALP after PTX

\begin{tabular}{lllll}
\hline Time & Calcium $(\mathrm{mmol} / \mathrm{L})$ & Phosphorus $(\mathrm{mmol} / \mathrm{L})$ & iPTH $(\mathrm{pg} / \mathrm{L})$ & ALP $(\mathrm{U} / \mathrm{L})$ \\
\hline Pre-op & $2.28 \mathrm{SD} 0.14$ & $2.59 \mathrm{SD} 0.42$ & $1327.12(956.5,1382.5)$ & $284.71 \mathrm{SD} 306.33$ \\
1 week post-op & $1.88 \mathrm{SD} 0.16$ & $2.03 \mathrm{SD} 0.43$ & $3.2(1.2,11.3) \Delta$ & $241.14 \mathrm{SD} 246.29$ \\
3 months post-op & $1.71 \mathrm{SD} 0.20$ & $2.11 \mathrm{SD} 0.57$ & $4.8(2.2,10.0) \Delta$ & $183.85 \mathrm{SD} 202.81$ \\
6 months post-op & $1.58 \mathrm{SD} 0.24$ & $2.23 \mathrm{SD} 0.59$ & $2.8(1.1,7.9) \Delta$ & $199.71 \mathrm{SD} 59.42$ \\
12 months post-op & $1.70 \mathrm{SD} 0.22$ & $2.11 \mathrm{SD} 0.29$ & $2.3(1.8,6.7) \Delta$ & $169.714 \mathrm{SD}$ 42.62 \\
$P$ value & $<0.05$ & $<0.05$ & $<0.001$ & $>0.05$ \\
\hline
\end{tabular}

The measurement data of normal distribution is represented by mean + standard deviation, and the measurement data of non-normal distribution is represented by $M(1 / 4,3 / 4)$. When the measurement data were consistent with the homogeneity of variance, used paired $t$-test for comparison between groups. Compared with preoperative, $* P<0.05, \Delta P<0.001$ 
glomerulonephritis. We searched the literature at home and abroad and found 31 cases of UTC. There were 15 cases with glomerulonephritis as the primary disease, accounting for $58.9 \%$ of patients. Owing to the small number of cases, we cannot confirm the correlation between UTC and primary glomerulonephritis.

Currently, the main treatment options include medication (calcium phosphate binders, calcimimetics, and sodium thiosulfate), surgical resection of the tumour, PTX, and renal transplantation, but there have been no large-scale controlled studies on the optimal treatment modalities [17]. In previous case reports, PTX was considered an effective treatment for UTC, and metastatic calcinosis was completely absorbed 1-5 months after PTX [18, 19]. Zhou et al. [20] reported a case of PD complicated with UTC that was completely absorbed 3 months after PTX with forearm transplantation. Daisuke et al. [21] presented a case of parathyroid carcinoma partially controlled by cinacalcet, in which the tumoral calcinosis was successfully resolved 1 year after a total PTX. However, Jaeger et al. [22] reported that, among three patients who received PTX treatment due to SHPT with UTC, only one had a tumour that could be absorbed after surgery. The other two patients had low preoperative serum ALP levels, and the tumours showed no significant absorption after surgery. The authors concluded that PTX is usually effective only in dialysis patients with severe hyperparathyroidism and high serum ALP levels. Wang et al. [23] retrospectively analysed the clinical data of eight UTC patients treated with PTX and found that five showed complete tumour absorption within 1-6 months after surgery, while the other four showed no significant tumour absorption. They reported that the PTX effectiveness rate for the treatment of UTC was $62.5 \%$ and that the non-absorption of UTC after PTX was related to a history of lower ALP levels and UTC. Zhang et al. [24] believed that prolonged persistence of UTC induced more fibroblasts and collagen encasing around calcium hydroxyapatite crystals, and this was one of the reasons why the calcinosis was difficult to absorb after PTX. Bas et al. [25] found that macrophages were associated with mineralised resorption and those macrophages could transform into osteoclasts and degrade calcified elastin. In the present study, the preoperative ALP level was significantly lower in the patient who did not experience UTC absorption after PTX than in the other seven patients, and this result is consistent with those of Wang et al. [23]. However, those study results were controversial because of the small sample size. Experimental verification with large sample studies is required to determine whether there is a relationship between low preoperative ALP and UTC nonabsorption after PTX. The mechanism of UTC absorption after PTX has not been elucidated, and it may be related to the ALP level and improvement of calcium and phosphorus metabolism after PTX. Serum calcium level and phosphate were decreased post-PTX, which then prevented the increase of tumour-like metastatic calcification [26]. Finally, hydroxyapatite and calcium pyrophosphate dihydrate crystals were dissolved. However, specific mechanisms of tumour absorption have not been reported. In our study, all eight patients with SHPT underwent PTX, of which seven showed complete UTC absorption within 1-6 months after PTX. Even for the patient whose surgery was unsuccessful, the tumour was completely absorbed 3 months after surgery. This confirmed the hypothesis that PTX can promote UTC absorption by improving calcium and phosphorus metabolism.

Regarding study limitations, first, this study followed a single-centre design, and due to the rarity of tumoral calcinosis in uremic SHPT patients, the number of patients in the study group was limited. Second, two patients failed to return to our hospital on time, and complete imaging data were not collected. Third, no experiments were performed to clarify mechanism of UTC absorption. In conclusion, this study shows the effectiveness of parathyroidectomy for the treatment of uraemic tumoral calcinosis. Thus, additional clinical studies with large sample sizes are needed to verify the effect of parathyroidectomy on uraemic tumoral calcinosis and to investigate the mechanism of uraemic tumoral calcinosis absorption after parathyroidectomy.

Author Contribution Haiting Huang, Jun Lu, Pengwei Guo, Jun Pang, Jing Ma, and Linlin He carried out the experimental work and conceived the study and participated in its design and coordination. Haiting Huang and Yanwu You drafted the manuscript. All authors read and approved the final manuscript.

Funding This study was supported by the Natural Science Foundation of Guangxi (No. 2019GXNSFBA245090).

Data Availability All data generated or analysed during this study are included in this published article.

\section{Declarations}

Ethical Approval and Consent to Participate This study was approved by human research ethics committee of the Affiliated Hospital of Youjiang Medical College for Nationalities (YYFY-LL-2019-004). All procedures performed in this study involving human participants were in accordance with the 1964 Helsinki Declaration and its later amendments or comparable ethical standards. The informed consent was obtained from all the participants.

Consent for Publication Written informed consent was obtained from all participants included in the study.

Competing Interests The authors declare no competing interest.

Informed Consent Written informed consent was obtained from all participants included in the study. 
Open Access This article is licensed under a Creative Commons Attribution 4.0 International License, which permits use, sharing, adaptation, distribution and reproduction in any medium or format, as long as you give appropriate credit to the original author(s) and the source, provide a link to the Creative Commons licence, and indicate if changes were made. The images or other third party material in this article are included in the article's Creative Commons licence, unless indicated otherwise in a credit line to the material. If material is not included in the article's Creative Commons licence and your intended use is not permitted by statutory regulation or exceeds the permitted use, you will need to obtain permission directly from the copyright holder. To view a copy of this licence, visit http://creativecommons.org/licenses/by/4.0/.

\section{References}

1. Fathi I, Sakr M (2014) Review of tumoural calcinosis: a rare clinico-pathological entity. World J Clin Cases 2:409-414. https://doi.org/10.12998/wjcc.v2.i9.409

2. Westermann L, Isbell LK, Breitenfeldt MK, Arnold F, Röthele E, Schneider J, Widmeier E (2019) Recuperation of severe tumoral calcinosis in a dialysis patient: a case report. World J Clin Cases 7:4004-4010. https://doi.org/10.12998/wjcc.v7.i23. 4004

3. Kamar FB, Mann B, Kline G (2016) Sudden onset of parathyroid hormone-independent severe hypercalcemia from reversal of tumoral calcinosis in a dialysis patient. BMC Nephrol 17:137. https://doi.org/10.1186/s12882-016-0355-y

4. Chu HY, Chu P, Lin YF, Chou HK, Lin SH (2011) Uremic tumoral calcinosis in patients on peritoneal dialysis: clinical, radiologic, and laboratory features. Perit Dial Int 31:430-439. https://doi.org/10.3747/pdi.2009.00250

5. Fukuta T, Tanaka T, Hashimoto Y, Omura H (2018) The relationship between multiple myeloma with renal failure and metastatic calcification. Case Rep Hematol 2018:7819792. https:// doi.org/10.1155/2018/7819792

6. Ke G, Li S, Cui Y, Chen X, Che H, Dou C, Lian Z, Zhang L, Li Z, Ma J, Feng Z, Yang J, Hu Y, Wang Y, Zhang H, Huang H, Su H, Guo J, He C, Liang X, Shi W, Ge P, Liu S (2020) Treatment of uremic tumoral calcinosis in maintenance hemodialysis patients. Blood Purif 49:658-664. https://doi.org/10.1159/ 000506115 (Epub 2020 Apr 14)

7. Guo R, Kurata T, Kondo T, Imanishi T, Mizuno T, Sakakibara T, Kasai Y (2017) Tumoral calcinosis in the cervical spine: a case report and review of the literature. J Med Case Rep 11:304. https://doi.org/10.1186/s13256-017-1474-1

8. Kim SM, Long J, Montez-Rath ME, Leonard MB, Norton JA, Chertow GM (2016) Rates and outcomes of parathyroidectomy for secondary hyperparathyroidism in the United States. Clin J Am Soc Nephrol 11:1260-1267. https://doi.org/10.2215/CJN.10370915

9. Mageau A, Guigonis V, Ratzimbasafy V, Bardin T, Richette P, Urena P, Ea HK (2017) Intravenous sodium thiosulfate for treating tumoral calcinosis associated with systemic disorders: report of four cases. Joint Bone Spine 84:341-344. https://doi. org/10.1016/j.jbspin.2016.10.009

10. Georges S, Srour N (2016) Metastatic pulmonary calcification in end-stage renal failure. CMAJ 188:E394. https://doi.org/10. 1503/cmaj. 150778

11. Ichikawa S, Baujat G, Seyahi A, Garoufali AG, Imel EA, Padgett LR, Austin AM, Sorenson AH, Pejin Z, Topouchian V, Quartier P, Cormier-Daire V, Dechaux M, Malandrinou FCh, Singhellakis PN, Le Merrer M, Econs MJ (2010) Clinical variability of familial tumoral calcinosis caused by novel GALNT3 mutations. Am J Med Genet A 152A:896-903. https://doi.org/ 10.1002/ajmg.a.33337
12. Nakamura T, Hirakawa K, Takaoka H, Iyama K (2016) Dystrophic calcinosis with both a huge calcified mass in the cervical spine and calcification in the chest wall in a patient with rheumatoid overlap syndrome. Clin Rheumatol 35:1403-1409. https://doi.org/10.1007/s10067-014-2696-x

13. Hocaoğlu N, Murat N, Mıcılı SC, Aydın B, Ergür BU, Kalkan Ş (2016) Correlation between amitriptyline-induced cardiotoxic effects and cardiac S100b protein in isolated rat hearts. Balk Med J 33:681-687. https://doi.org/10.5152/balkanmedj.2016.150435

14. Vieira AR, Lee M, Vairo F, LoguercioLeite JC, Munerato MC, Visioli F, D'Ávila SR, Wang SK, Choi M, Simmer JP, Hu JC (2015) Root anomalies and dentin dysplasia in autosomal recessive hyperphosphatemic familial tumoral calcinosis (HFTC). Oral Surg Oral Med Oral Pathol Oral Radiol 120:e235-e239. https://doi.org/10.1016/j.oooo.2015.05.006

15. Rui H, Zhao Y, Liu S et al (2006) A case report of huge metastatic calcification during the whole pulse therapy of rocalcium. China Blood Purif 11:782.Chinese

16. Binnani P, Aggarwal V, Bahadur MM, Fulara N (2008) Tumoral calcinosis (Teutschlander disease) in a dialysis patient. Indian $\mathbf{J}$ Nephrol 18(3):122-124

17. Ando T, Mochizuki Y, Iwata T, Nishikido M, Shimazaki T, Furumoto A, Minami S, Kinoshita N, Kawakami A (2013) Aggressive pulmonary calcification developed after living donor kidney transplantation in a patient with primary hyperparathyroidism. Transplant Proc 45:2825-2830. https://doi.org/10.1016/j.trans proceed.2013.01.071

18. Verma S, Bassily E, Jarmi T (2017) An extreme case of tumoral calcinosis in an end-stage renal disease patient. Kidney Int 91:991. https://doi.org/10.1016/j.kint.2016.10.031

19. Xiong M, Wang J, Li XD, Yu YW, Zhang L (2020) Clinical characteristics of 10 patients with uremic tumoral calcinosis. Zhonghua Nei Ke Za Zhi 59:860-865 Chinese. https://doi.org/10.3760/ cma.j.cn112138-20200305-00193

20. Zhou H, Yang M, Zou Y (2018) Uraemic tumoral calcinosis in a peritoneal dialysis patient. Intern Med J 48:1544-1546. https:// doi.org/10.1111/imj.14130

21. Takada D, Tsukamoto T, Fuse M, Kada S, Yanagita M (2017) The use of Cinacalcet hinders the diagnosis of parathyroid carcinoma in a chronic dialysis patient: a case report. BMC Nephrol 18:315. https://doi.org/10.1186/s12882-017-0733-0

22. Jaeger VA, Newman MG, Mirkes CR (2017) Metastatic calcinosis cutis in end-stage renal disease. Proc (Bayl Univ Med Cent) 30:368-369. https://doi.org/10.1080/08998280.2017.11929652

23. Wang J, Zeng M, Yang G, Huang Y, Wu B, Guo J, Wang N, Xing C (2019) Effects of parathyroidectomy on tumoral calcinosis in uremic patients with secondary hyperparathyroidism. BMC Surg 19:133. https://doi.org/10.1186/s12893-019-0603-8

24. Zhang R, Li G, Yang L, Li Y, Ou J, Zhang D, Chen T, Feng S (2016) Multiple ectopic calcifications in subcutaneous tissues with chronic renal failure: a case report. Int J Surg Case Rep 29:113119. https://doi.org/10.1016/j.ijscr.2016.09.041

25. Bas A, Lopez I, Perez J, Rodriguez M, Aguilera-Tejero E (2006) Reversibility of calcitriol-induced medial artery calcification in rats with intact renal function. J Bone Miner Res 21:484-490. https://doi.org/10.1359/JBMR.051211

26. Slavin RE, Wen J, Barmada A (2012) Tumoral calcinosis-a pathogenetic overview: a histological and ultrastructural study with a report of two new cases, one in infancy. Int J Surg Pathol 20:462-473. https://doi.org/10.1177/1066896912444925

Publisher's Note Springer Nature remains neutral with regard to jurisdictional claims in published maps and institutional affiliations. 